\title{
Sevoflurane anesthesia: impact on postoperative cognitive dysfunction
}

\author{
Victoria Rusu \\ Department of Anesthesiology and Resuscitation No 2 \\ Nicolae Testemitanu State University of Medicine and Pharmacy, Chisinau, the Republic of Moldova \\ Author's ORCID iD, academic degrees and contribution are available at the end of the article \\ Corresponding author - Victoria Rusu, e-mail: victoria_rusu@mail.ru \\ Manuscript received November 01, 2021; revised manuscript December 10, 2021; published online December 17, 2021
}

\begin{abstract}
Background: Sevoflurane is the inhalational anesthetic agent that is used widely in operating room. It is currently the most commonly used inhalation anesthetic in operating rooms. A series of studies on animal and human model detected the association of intraoperative use of sevoflurane and postoperative cognitive dysfunction (POCD) manifestation. On the other hand other studies demonstrate the same POCD associated with intravenous agents. Relevant multicentric trials got the reasons to suspect other key factors in developing postoperative cognitive dysfunction.

Conclusions: The intra-anesthetic use of sevoflurane has been associated for a long time with the higher incidence of POCD. The mechanism was not identified, and the theory of neuroinflammation remained the main key of pathophysiological reaction that leads to cognitive dysfunction. Recent multicentre trial gives reliable information that the use of intravenous anesthetic agents is associated with the same POCD. Neuroinflammation remains to be the mediator of cognitive disorders, and apparently IL- 6 keeps a major role in them. Future studies are needed to be conducted to identify the role of anesthetic agents in determining the neuroinflammation.

Key words: sevoflurane, propofol, outcome, postoperative cognitive dysfunction.
\end{abstract}

Cite this article

Rusu V. Sevoflurane anesthesia: impact on postoperative cognitive dysfunction. Mold Med J. 2021;64(6):49-52. https://doi.org/10.52418/moldovanmed-j.64-6.21.09.

\section{Introduction}

Inhalational anesthetic agents have a long lasting history, from the beginning of general anesthesia and the discovery of ether in 1866 , and our days where we can use a large variety of inhalational agents: desflurane, sevoflurane, isoflurane and others, to provide a safe general anesthesia. Despite large use of them, worldwide there are still many questions remain to be clarified concerning the use of inhalation anesthesia and its effects on the recovery of the patients. Sevoflurane is one of the most widely used anesthetics for the induction and maintenance of general anesthesia. Despite the large use, however, its anesthetic mechanism remains unclear like for the rest of the agents. Sevoflurane reportedly causes amnesia, analgesia, coma, and quiescence, primarily by inhibiting N-methil-D-aspartat (NMDA) receptors. In addition, in vivo studies have suggested that gamma-aminobutyric acid (GABA) type A (GABAA) receptors, nicotinic acetylcholine receptors, and voltage-gated sodium channels are potential targets for sevoflurane related to its hypnotic effects.

The clinical use of the sevoflurane extended in countries from Eastern Europe as well, for the last 10 years. It is currently the most commonly used inhalation anesthetic in operating rooms. It has been demonstrated excellent respiratory tolerance in different clinical cases, as well as the hemodynamic stability in cardiopulmonary bypass, providing a safe anesthetic process for different categories of surgery from one-day surgery to complex long-lasting surgery.

\section{Material and methods}

There was made a scientific review of the latest articles from PubMed database that were published between 20162021, concerning sevoflurane and its effects. The selection criteria were: prospective studies, guidelines, trials and meta-analyses. Key words used in search: "sevoflurane", "propofol", "outcome", "postoperative cognitive dysfunction" (POCD). The articles contain a detailed analysis and synthesis of the recommendations, concerning the use of the sevoflurane and cognitive disfunction after its use. There were selected articles, taking into consideration their title, and selecting abstracts. In the process of searching by title using key words, there have been found 686 results. After selecting the period of years 2016-2021, 352 results were found.

\section{Results}

One of the most frequent postanesthetic complications, especially in elderly patients, after the use of the sevoflurane is POCD, which is a multifactorial, neurodegenerative condition, mechanisms of which remain unclear. Many studies and repeated clinical trials have shown that after exposure to sevoflurane-inhalation anesthesia, humans and animals 
experience varying degrees of cognitive dysfunction [1, 2]. Different hypotheses like neuroinflammation, changes in neurotransmitters, a decrease in brain-derived neurotrophic factor (BDNF), mitochondrial oxidative stress, and changes in $A \beta$ concentrations regarding the pathogenesis of sevoflurane-induced POCD, are described by researchers. Still these mechanisms are not completely independent and interact with each other, finally resulting in POCD. In recent years, increasing attention has been paid to the relationship between sevoflurane-induced POCD and neuroinflammation, changes in neurotransmitters, and BDNF reduction [3]. Alalawi R. and Yasmeen N. in 2018 showed that the incidence of POCD was at least twice as high in individuals older than 60 years as compared to younger age groups [4]. The high incidence of POCD in older patients may be related to specific susceptibility factors. Aging itself is a risk factor for cardiovascular, respiratory, renal, and neurodegenerative diseases. Immune responses also decrease with age. Also, the pharmacokinetics and pharmacodynamics of older patients are considerably altered compared to younger patients. Sevoflurane-based anesthesia in older patients results in a lower minimum alveolar concentration of gas and an increased cumulative effect of it, with gradual degeneration of various organ functions [4]. Hence, sevoflurane may remain in the blood for longer periods in older than in younger patients after anesthesia [5]. The fragile balance between neuroinflammation and neuronal functioning in older patients is easily interrupted upon pathological invasion [6, 7]. In 2015 Qiao Y. et al., have found elevated plasma concentrations of S- $100 \beta$ protein, TNF- $\alpha$, and IL- 6 in patients receiving sevoflurane anesthesia [7]. Other animal study in 2020, headed by Yang L. et al., determined that sevoflurane has been found to induce increased inflammation and apoptosis of hippocampal neurons in older rats [6]. Sevoflurane-induced anesthesia disturbs the balance between neuronal functioning and neuroinflammation, especially in older patients, increasing the incidence of POCD. To reduce the incidence of sevoflurane-induced POCD the optimal management of pre-existing diseases and maintenance of optimum functioning of the nouronal homeosthasis may be an effective way. Another randomized controlled trial of sevoflurane-induced anesthesia in laparoscopic cholecystectomy, showed aggravated POCD compared to propofol group of patients after laparoscopic cholecystectomy [8]. On the other hand, some studies have confirmed that sevoflurane exerts neuroprotective effects through specific pathways, in a rat model of focal cerebral ischemia [9]. Recently in 2020, Lu G. et al., presented the results of an animal model study where sevoflurane pre-treatment exerted a neuroprotective effect by reducing signaling activity and activating autophagy [10]. Also, sevoflurane post-treatment can regulate mir-203 expression to attenuate cerebral ischemia - reperfusion-induced neuroinflammation by targeting MyD88 [11].

Neuroinflammation is one of the multifactorial determinants, particularly in the hippocampus, that has been shown to play an important role in POCD. Block et al., in 2007, then Lu B. et al., in 2020 showed in their studies that the activation of microglia may play the most important role in the development of POCD, as inflammation of microglia is recognized as the main source of pro-inflammatory cytokines and chemokines in the central nervous system (CNS) $[12,13]$. Another study headed by Su W. et al., in 2020 is concerning neuroinflammation and microglial activation trigger that amplify a complex cascade of reactions, including immune response activation, microcirculatory changes, increased hippocampal oxidative stress, and increased blood-brain barrier permeability [14]. Other studies that researched the pro-inflammatory cytokines IL-1 $\beta$, IL-6, and TNF- $\alpha$ determined that they were statistically significantly increased in the brains of rats exposed to sevoflurane in a number of studies [8].

Zhu X. et al., in their study from 2021 showed the correlation between the property of sevoflurane to be highly and lightly diffused across the cell membrane without binding to specific receptors on the cell membrane and to direct stimulation of NF- $\kappa B$ signaling. Sevoflurane has been shown to increase intracellular $\mathrm{Ca} 2+$ by activating GABA receptors, inducing mitochondrial damage, and increasing the levels of intracellular reactive oxygen species (ROS) [15]. A sevoflurane-induced increase in intracellular $\mathrm{Ca} 2+$ may activate NF- $\kappa \mathrm{B}$ signaling and lead to increased concentrations of pro-inflammatory cytokines. IL-17A, a novel cytokine, increases statistically significantly in the hippocampus of sevoflurane-induced aged rats and can promote the binding of Act1 (an activator of NF- $\kappa B$ ) and IL-17R to induce activation of the NF- $\kappa B$ signaling pathway [15].

Neuroinflammation is a critical neuropathological process for postoperative cognitive dysfunction. This finding is mostly based on results from animal studies. It has been shown that surgery on peripheral tissues causes systemic inflammation that then induces neuroinflammation in rodents $[16,17]$. Consistent with this finding, serum interleukin- 6 concentrations were increased after surgery in patients with or without delayed neurocognitive recovery, but the interleukin- 6 concentrations were higher in patients with delayed neurocognitive recovery than that in patients without delayed neurocognitive recovery, providing initial evidence that heightened inflammation may be a pathologic process for delayed neurocognitive recovery in humans. There were no changes in the serum interleukin$1 \beta$, interleukin-10, and tumor necrosis factor- $\alpha$ within 24 $\mathrm{h}$ after the surgery. These results suggest that only selected cytokines, like interleukin-6 in this surgical population, are induced after surgery. Interestingly, the serum concentrations of vascular endothelial growth factor, intercellular adhesion molecule, transforming growth factor- $\beta 1, C 3 \alpha$, and advanced glycation end products were first decreased and then recovered after surgery. The first four factors can modulate immune functions. Advanced glycation end products are an oxidative stress marker. The reasons for this pattern of change are not known but may indicate a decreased immune function at the end of surgery, which recovers with time after surgery [18]. 
A multicenter, randomized trial on intravenous versus volatile anesthetic effects on postoperative cognition in elderly patients undergoing laparoscopic abdominal surgery, published in 2021, showed that patients with laparoscopic abdominal surgery under propofol-based anesthesia had a delayed neurocognitive recovery incidence similar to that of patients under sevoflurane-based anesthesia. This recent study determines clinicians to take into consideration that anesthetic choice may not be a decisive factor for delayed neurocognitive recovery [8]. That study has identified the fact that a high concentration in serum interleukin- 6 is an independent risk factor for delayed neurocognitive recovery, and this is a marker that should be taken into consideration regarding patients with risk to develop POCD. This finding provides clinical evidence for the role of inflammation in delayed neurocognitive recovery.

In the sevoflurane model studies, calcium influx and increased exposure to ROS can lead to the activation of the NLRP3 inflammasome, which may influence the neurological outcome in postoperative period (fig. 1).

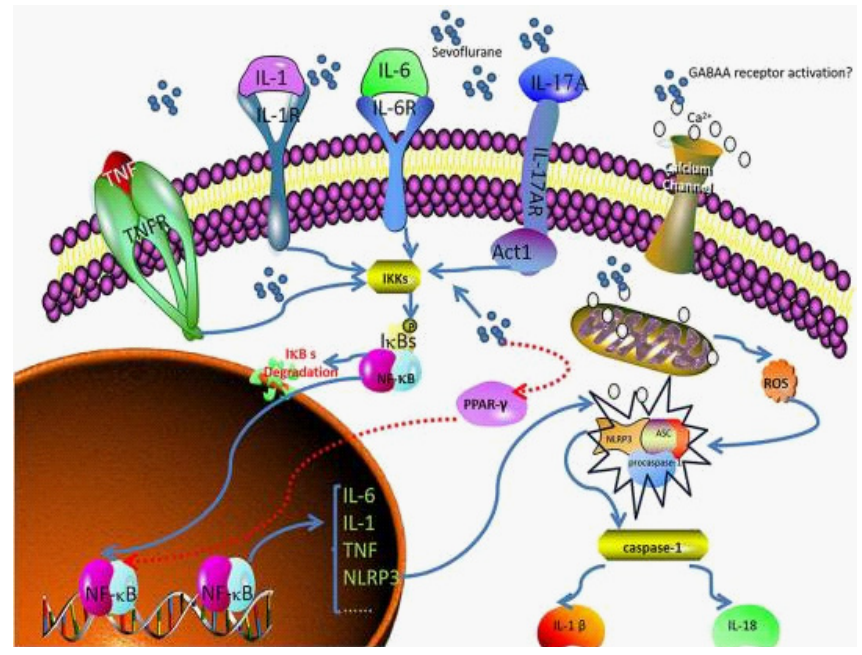

Fig. 1. Sevoflurane induces neuroinflammation and leads to POCD [8]

The damage of mitochondria and exposure to mitochondrial contents, such as ROS, are essential for the assembly of the NLRP3 inflammasome. Also is required calcium influx for optimal activation of the NLRP3 inflammasome [8].

Dong P. et al. in their study in 2018, confirmed as well that sevoflurane could contribute to inducing the neuroinflammation mediated by microglia by downregulating hippocampal PPAR- $\gamma$, exacerbating cognitive dysfunction [19]. The theory of neuroinflammation, has more power to demonstrate the incidence of POCD after sevoflurane model anesthesia since peroxisome proliferator-activated receptor- $\gamma$ (PPAR- $\gamma$ ) is a ligand-inducible transcription factor of the nuclear hormone receptor family and is expressed in several cell types in the brain, including microglia, astrocytes, and neurons. The Nod-like receptor protein 3 (NLRP3) inflammasome is determinant to the immune response, and includes NLRP3, apoptosis-associated speck-like protein containing a caspase-recruitment domain, and procaspase- 1 .

\section{Discussion}

In recent years, many studies provided information considering the relationship between sevoflurane-induced POCD and neuroinflammation, changes in neurotransmitters, and BDNF reduction.

Postoperative cognitive disfunction is a multifactorial, neurodegenerative condition mechanisms of which remain unclear due to many studies conducted for the last 5 years. Several clinical trials, as well as many animal studies have shown that after sevoflurane-inhalation anesthesia, humans and animals experience different degrees of cognitive disfunction. Hypotheses regarding the pathogenesis of sevoflurane-induced POCD, including neuroinflammation, changes in neurotransmitters, decrease in BDNF, mitochondrial oxidative stress, and changes in $\mathrm{A} \beta$ concentrations, couldn't give a complete answer to the question of correlation between sevoflurane anesthesia and POCD. The answer is more difficult to get as long mechanisms are not completely independent and they interact with each other.

The importance of patient-related factors, such as age, cognitive status before the surgery, may also affect the incidence of POCD, being related or not to the choice of the anesthetic agent, shows a recent multicenter randomized trial [20]. Related studies that confirm the correlation between sevoflurane anesthesia and neuroinflammation, exacerbating that way cognitive dysfunction, recent randomized trials show the same cognitive dysfunction being relative to intravenous anesthesia agents. Neuroinflammation still remains the key factor of POCD and its correlation or not with the selection of anesthetic agent was not determined.

Regarding the limitation of studies, it was determined by the American Society of Anesthesiology that the physical status classification, mini-mental status examination scores, and duration of hospitalization were predictors for delayed neurocognitive recovery. Regardless of the type of anesthetic agent, a high blood interleukin- 6 concentration in postoperative period is a risk factor for developing delayed neurocognitive recovery. In fact, interleukin- 6 concentration in the blood at this time is the only independent risk factor for delayed neurocognitive recovery as determined by multiple studies [21, 22]. However, its concentration as a risk factor for delayed neurocognitive recovery has not been reported.

The role of intravenous and volatile anesthetics in postoperative cognitive dysfunction in humans remains unclear. Therefore, future research should be aimed at further elucidating the pathogenesis of induced POCD and developing an effective combination therapy to minimize the neuroinflammation and protective strategies for minimizing POCD, and the length of stay in hospitals.

\section{Conclusions}

The intra-anesthetic use of sevoflurane has been associated for a long time with the higher incidence of POCD. The mechanism was not identified, and the theory of neuroinflammation remained the main key of pathophysiological reaction that leads to cognitive dysfunction. Recent multi- 
centric trial gives reliable information that the use of intravenous anesthetic agents is associated with the same POCD. Neuroinflammation remains to be the mediator of cognitive disorders, and apparently IL-6 keeps a major role in them. Future studies are needed to be conducted to identify the role of anesthetic agents in determining the neuroinflammation.

\section{References}

1. Cabrera OH, Tesic V, Tat QL, Chastain S, Quillinan N, JevtovicTodorovic V. Sevoflurane-induced dysregulation of cation-chloride cotransporters NKCC1 and KCC2 in neonatal mouse brain. Mol Neurobiol. 2020;57(1):1-10. doi: 10.1007/s12035-019-01751-1.

2. Cai Y, Huang P, Xie Y. Effects of huperzine A on hippocampal inflammatory response and neurotrophic factors in aged rats after anesthesia. Acta Cir Bras. 2020;34(12):e201901205. doi: 10.1590/s0102865020190120000005 .

3. Afonina IS, Zhong Z, Karin M, Beyaert R. Limiting inflammation - the negative regulation of NF- $\mathrm{KB}$ and the NLRP3 inflammasome. Nat Immunol. 2017;18(8)861-869. doi: 10.1038/ni.3772.

4. Alalawi R, Yasmeen N. Postoperative cognitive dysfunction in the elderly: a review comparing the effects of desflurane and sevflurane. J Perianesth Nurs. 2018;33(5):732-740. doi: 10.1016/j.jopan.2017.04.009.

5. Araki R, Hayashi K, Sawa T. Dopamine D2-receptor antagonist droperidol deepens sevoflurane anesthesia. Anesthesiology. 2018;128(4):754763. doi: 10.1097/ALN.0000000000002046.

6. Yang LH, Xu YC, Zhang W. Neuroprotective effect of CTRP3 overexpression against sevoflurane anesthesia-induced cognitive dysfunction in aged rats through activating AMPK/SIRT1 and PI3K/AKT signaling pathways. Eur Rev Med Pharmacol Sci. 2020;24(9):5091-5100. doi: 10.26355/eurrev_202005_21202.

7. Qiao Y, Feng H, Zhao T, Yan H, Zhang H. Postoperative cognitive dysfunction after inhalational anesthesia in elderly patients undergoing major surgery: the influence of anesthetic technique, cerebral injury and systemic inflammation. BMC Anesthesiology. 2015;15:154. 23. doi: 10.1186/s12871-015-0130-9.

8. Wang CM, Chen WC, Zhang Y, Lin S, He HF. Update on the mechanism and treatment of sevoflurane-induced postoperative cognitive dysfunction. Front Aging Neurosci. 2021;13:702231. doi: 10.3389/ fnagi.2021.702231.

9. Belrose JC, Noppens RR. Anesthesiology and cognitive impairment: a narrative review of current clinical literature. BMC Anesthesiol. 2019;19(1):241. doi: 10.1186/s12871-019-0903-7.

10. Lu G, Rao D, Zhou M, Liangpu Xu, Zhang L, Zhang S, Wang Y. Autophagic network analysis of the dual effect of sevoflurane on neurons associated with GABARAPL 1 and 2. Biomed Res Int. 2020;2020:1587214. doi: $10.1155 / 2020 / 1587214$.
11. Zhong H, Chen H, Gu C. Sevoflurane post-treatment upregulated miR-203 expression to attenuate cerebral ischemia-reperfusion-induced neuroinflammation by targeting MyD88. Inflammation. 2020;43(2):651663. doi: 10.1007/s10753-019-01147-2.

12. Block ML, Zecca L, Hong JS. Microglia-mediated neurotoxicity: uncovering the molecular mechanisms. Nat Rev Neurosci. 2007;8(1):57-69.

13. Lu B, Yuan H, Zhai X, Li X, Qin J, Chen J, Meng B. High-pressure pneumoperitoneum aggravates surgery-induced neuroinflammation and cognitive dysfunction in aged mice. Mediators Inflamm. 2020;2020:6983193. doi: 10.1155/2020/6983193.

14. Su W, Xie M, Li Y, Gong X, Li J. Topiramate reverses physiological and behavioral alterations by postoperative cognitive dysfunction in rat model through inhibiting TNF signaling pathway. Neuromolecular Med. 2020 Jun;22(2):227-238. doi: 10.1007/s12017-019-08578-y.

15. Zhu X, Yao Y, Guo M, Li J, Yang P, Xu H, Lin D. Sevoflurane increases intracellular calcium to induce mitochondrial injury and neuroapoptosis. Toxicol Lett. 2021 Jan 1;336:11-20. doi: 10.1016/j.toxlet.2020.11.002.

16. Butz M, El Shazly J, Sammer G, Tschernatsch M, Kastaun S, Yenigün $\mathrm{M}$, et al. Decreasing postoperative cognitive deficits after heart surgery: protocol for a randomized controlled trial on cognitive training. Trials. 2019;20(1):733. doi: 10.1186/s13063-019-3799-0.

17. Chen H, Wu X, Gu X, Zhou Y, Ye L, Zhang K, et al. Tacrine (10)-hupyridone prevents post-operative cognitive dysfunction via the activation of BDNF pathway and the inhibition of AChE in aged mice. Front Cell Neurosci. 2018;12:396. doi: 10.3389/fncel.2018.00396.

18. Chen R, Zhang T, Kuang L, Chen Z, Ran D, Niu Y, et al. Cholinergic synaptic transmissions were altered after single sevoflurane exposure in Drosophila pupa. Biomed Res Int. 2015;2015:485709. doi: $10.1155 / 2015 / 485709$

19. Dong P, Zhao J, Li N, Lu L, Li L, Zhang X, et al. Sevoflurane exaggerates cognitive decline in a rat model of chronic intermittent hypoxia by aggravating microglia-mediated neuroinflammation via downregulation of PPAR- $\gamma$ in the hippocampus. Behav Brain Res. 2018;347:325-331. doi: 10.1016/j.bbr.2018.03.031

20. Li Y, Chen D, Wang H, Wang Z, Song F, Li H, et al. Intravenous versus volatile anesthetic effects on postoperative cognition in elderly patients undergoing laparoscopic abdominal surgery. Anesthesiology. 2021;134(3):381-394. doi: 10.1097/ALN.0000000000003680.

21. Quan C, Chen J, Luo Y, Zhou L, He X, Liao Y, Chou J, Guo Q, Chen AF, Wen O. BIS-guided deep anesthesia decreases short-term postoperative cognitive dysfunction and peripheral inflammation in elderly patients undergoing abdominal surgery. Brain Behav. 2019;9(4):e01238. doi: 10.1002/brb3.1238.

22. Silbert BS, Scott DA, Evered LA, Lewis MS, Kalpokas M, Maruff P, Myles PS, Jamrozik K. A comparison of the effect of high- and low-dose fentanyl on the incidence of postoperative cognitive dysfunction after coronary artery bypass surgery in the elderly. Anesthesiology. 2006;104(6):113745. doi: 10.1097/00000542-200606000-00007.

\section{Author's ORCID iD and academic degrees}

Victoria Rusu, MD, PhD, Assistant Professor of Anesthesiology - https://orcid.org/0000-0002-5241-4067

\section{Author's contribution}

VR conceptualized the idea, conducted literature review, collected the data, interpreted the data, and wrote the manuscript.

\section{Funding}

This study was supported by Nicolae Testemitanu State University of Medicine and Pharmacy and the journal publication fee was covered by "UNIMED FARMA" SRL. The trial was the author's initiative. The author is independent and takes responsibility for the integrity of the data and accuracy of the data analysis.

\section{Ethics approval and consent to participate}

No approval was required for this study.

\section{Conflict of Interests}

The author has no conflict of interests to declare. 